\title{
Research on Agent-oriented distributed inventory management
}

\author{
Shuren ZHU \\ School of Information, Guangdong University of Finance \& Economics, Guangzhou, China \\ Zhusr@gdufe.edu.cn,
}

\begin{abstract}
Keywords: Artificial intelligence Agent; Agent-oriented Modeling; Distributed Inventory
Abstract. According to characteristics of artificial intelligence Agent of autonomy, sociality, responsiveness, using Agent oriented approach and extracting Agent based on the function demand of distributed inventory manage system, detailed analyzing the process of modeling through planning of single Agent, dependence of Agent, Agent interaction protocol description and structure definition of Agent, finally giving an UML class view of distributed inventory management system based on Agent. The model of agent system is a simulation system consists of a plurality of agents can be calculated, which takes a bottom-up approach.
\end{abstract}

\section{Introduction}

The traditional modeling method based on mathematical formula became more and more difficult to adapt to the complex system simulation, especially for those parts of the heterogeneous system[1-2]. With the development of software and hardware of computer, a modeling method based on object gradually replaced the traditional methods that relied on mathematic formulas, more and more scholars began to use object oriented method for the simulation of complex system[3]. In the 1970s, object oriented technology obtained smart extension, and the field of artificial intelligence stared using the concept of agent[4-5]. There are many common points between agent and object, the only difference is that the agent of artificial intelligence is a kind of computer system in a package under the certain conditions, and has its own special characters of autonomy, sociality, reactivity, mobility, cooperation[6]. Agent technology endowed with more humane on the basis of object oriented technology, and turned to be a kind of more advanced computer simulation[7-8]. This paper uses the agent oriented approach to describe the process of constructing the model of distributed inventory management system according to the characteristics of it, and gives the UML class view of distributed inventory management system.

\section{The design of distributed inventory management system}

Distributed inventory system is composed of a plurality of warehouse, it based on coordination center [9], which is shown as figure 1. In this system, when there is order demand and warehouse inventory is not enough orders, warehouse directly send request to the coordination center and apply for product, the information system of coordination center will first to get real-time stock information after query all warehouse, then choose an inventory has sufficient products and closest to the warehouse which is shortage of products to and send allocation orders[10].Using the distributed inventory management doesn't require each warehouse reach the level of safety inventory, as long as the overall inventories remain above safety inventory of system. When the overall inventories descend to the reorder point, each warehouse applies for a joint order to the coordination center. If there is only one warehouse's inventory decreases to a reorder point and the overall inventories doesn't fall to the overall inventories reorder point. The warehouses out of stock make mutual adjustment under the unified scheduling of coordinating center, thereby reducing the number of orders[11-12].

According to the characteristics of distributed inventory management system, the whole system can be divided into three levels of senior management layer, virtual dynamic cell layer, and executive layer (warehouse management).Therefore, distributed inventory management system based on agent can be 
also divided into three levels of senior management Agent, virtual dynamic management cell Agent and executive Agent. Details of each layer as follow:

Senior management Agent: Top management Agent is the top management layer during the process of inventory management, which is mainly responsible for the macro-control of the entire inventory system, including make decisions of overall safety stock quantity and ensure that the operation of system normal and reasonable.

virtual dynamic management cell Agent: Virtual dynamic management cell Agent is responsible to query inventory information of warehouses, optimize the use of internal resources according to the warehouse real inventory when receiving the request, and pass the information of each warehouse to senior management layer.

executive Agent: Execution Agent is in the bottom layer of the system, they realize the full use of resources and resource sharing through the virtual dynamic management cell Agent.

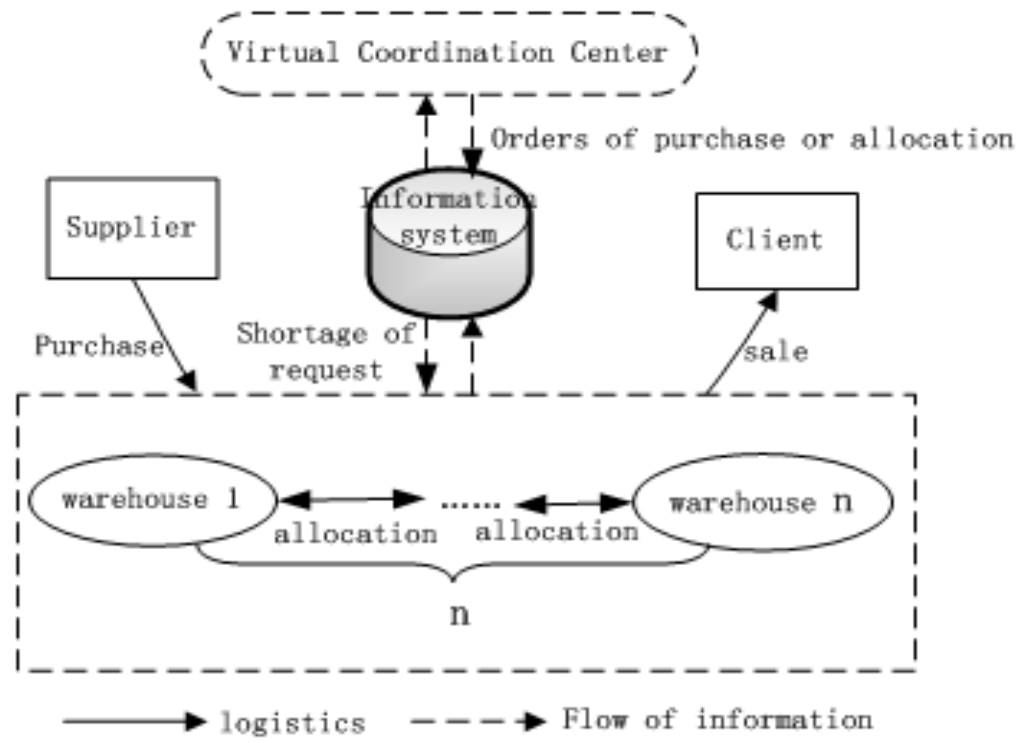

Figurel Distributed Inventory System Model

\section{Construction of distributed inventory management system model}

\section{Planning of single agent}

For a given system, planning a single Agent is abstract Agent from system. In general, the abstract process of Agent can start from the physical structure of the system, around the target of the system.In the distributed inventory management system, entities are isomorphic and can instantiated for the corresponding homogeneous Agent when the model of system under operated. However, it can't clear abstract all the functions of the system to a single Agent as the business of distributed inventory management system is various and there are many kinds of complex functions. It will increase the system complexity of organization and control if there is too much Agent. Therefore, the distributed inventory management system can be abstracted into the following Agent according to the main function of system, such as senior management Agent, virtual dynamic cell management Agent, warehouse management Agent, inventory management Agent, storehouse management Agent, stock-taker Agent and dispatcher Agent.

\section{Dependence of Agent}

After planning single Agent, it needs to establish architecture of Agent. If one Agent needs help of another Agent to achieve its goals, it is called a dependency exists between two Agent. The dependency relationship between Agent include independence, one-way dependency, two-way dependency and interactive dependency [1]. The detailed introduction as follows:

a. Independence: there is no dependency relationship exist between Agent.

b. one-way dependency: one Agent one-way depends on another Agent. 
c. two-way dependency: two Agent have mutual dependence for a common goal.

d. interactive dependence: the first Agent relies on the second Agent for a goal, and the second Agent also depends on the first Agent in order to achieve a certain goal.

By the planning of single Agent, we know that distributed inventory management system can be mainly abstracted of senior management Agent, virtual dynamic cell management Agent, warehouse management Agent, inventory management Agent, storehouse management Agent, stock-taker Agent and dispatcher Agent. The cases of warehouse management Agent contain registration daily management, warehouse entry management, audit accounting management. The cases of inventory management Agent contain products transfer management, inventory dispatch management. The cases of storehouse management Agent contain outbound order management, storehouse information management, audit of storehouse information management. The cases of senior management Agent contain report management, query management, basic data management, safety inventory management. The cases of virtual cell management Agent contain cell management. The cases of stock-taker Agent contain inventory list management, inventory profit and loss sheet management. The cases of dispatcher Agent contain delivery order management. Therefore, Agent can be combined to a set of use case packages based on the Agent oriented UML model. In other words, the underlying specific implementation of distributed inventory management system can be abstracted certain cases of package.

\section{INTERACTION PROTOCOL OF AGENT}

In order to realize the respective target of system effectively, each Agent must agree on the common target they focus on, and then take unified action. However, due to the difference of their goals, available resources and the status in the system, they need protocol to reach an agreement. The formulations of the FIPA98, FIPA2000 and other standards made by intelligent agent organization FIPA (Foundation for Intelligent Physical Agents) general illustrate static intelligent Agent and designate standards for language, content, communicate framework and protocol [3].

The interaction protocol In the process of inventory management that virtual cell management Agent sends dispatch tasks to inventory management Agent can be expressed as follows: Inventory management Agent send massage of checking inventory information to stock-taker Agent when it receives product application, and the stock-taker Agent transits the inventory information table of itself to Inventory management Agent if it was updated, otherwise, it sends non idle message. If the returned inventory quantity can't meet product application, inventory management Agent asks virtual cell management Agent for dispatching. When virtual cell management Agent received dispatch request, it sends massage of checking inventory information to the other inventory management Agent, and chooses an available warehouse to allocate dispatch task massage according to current inventory condition of all warehouses. When the warehouse receives dispatch task massage, the inventory management Agent transits it to storehouse management Agent, and then pass it to dispatcher Agent in order to realize the whole process of dispatch if the inventory information was updated, otherwise, inventory management Agent sends non idle message to virtual cell management Agent. After it receives non idle message, the virtual cell management Agent can cancel send dispatch task, it means that there is no available warehouse with enough inventory quantity closest to which is shortage of products.

\section{STRUCTURE DEFINITION OF AGENT}

As Agent oriented technology is an intelligent extension of object oriented technology, the structure of Agent has a lot in common with object in the process of model construction, such as the encapsulation, inheritance and polymorphism of data and methods. The only difference is the degree of autonomy. For example, in the JAVA programming language, if programmer declares an instance for the public, and if the instance method is invoked by other objects, these objects are ready for implementing this method at any time. Autonomous of object embodied in the control of the state, and their behavior can't reflect good autonomy. In the system model composed of Agent, the specific realization is different from object oriented. 
For example, virtual cell management Agent has behaviors (Methods) of viewing inventory information and sending dispatch tasks, the inventory management Agent has behaviors of returning inventory information and receiving dispatch task, Virtual cell management Agent has behavior of viewing inventory information. The inventory management Agent receives requirements of checking view inventory information sent from virtual cell management Agent and returns inventory information to the virtual cell management Agent. If the amount of inventory meets the demand for dispatch tasks, virtual cell management Agent sends dispatch tasks to inventory management Agent, otherwise it will not implement this behavior.

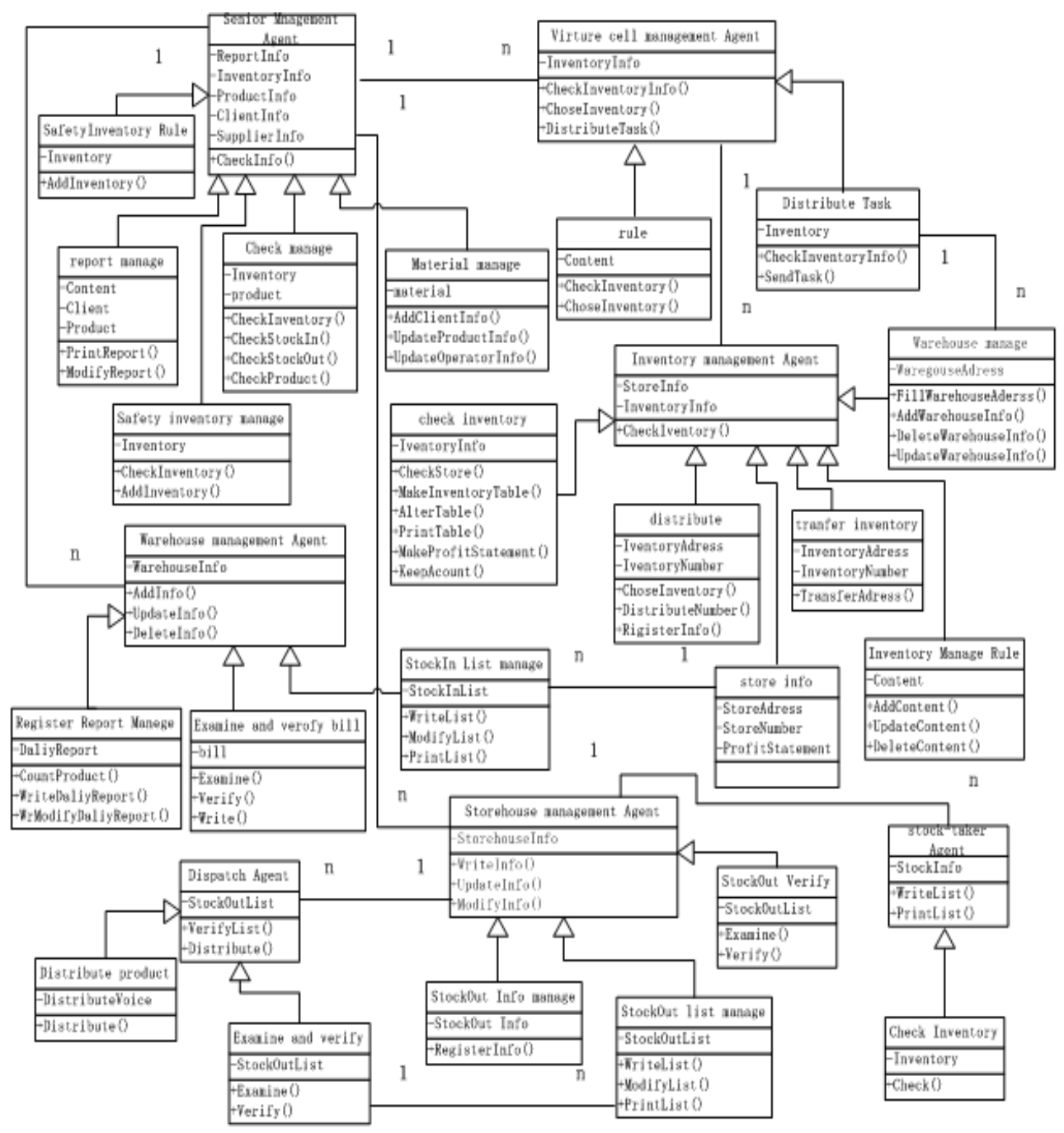

Figure6 The class view based on UML of Agent oriented distributed inventory management system

Therefore, it is not the same as object on the decision-making mechanism of whether to perform a certain behavior. In the object oriented case, decision made by object which uses method, while in the Agent oriented case, decision made by Agent that receive the request .

Although and there is obvious difference between Agent and object, the Agent oriented technology as an intelligent extension of object oriented technology gives more humanization feature on the basis of inherit all advantages of object oriented [5]. Therefore, it can still use object oriented technology to realize the definition of the structure of Agent, which uses class diagrams of UML to represent the structure of Agent.

In the use of class diagrams of UML to represent the structure of Agent, the features of individual in a practical system are mapped to attributes (the internal status) of Agent, the behaviors of individual are mapped to conduct rules (methods) of Agent, so as to get the structure model of Agent. Inventory manage Agent is a super class, and check inventory, distribute, transfer inventory, warehouse manage 
are behaviors (method) of Agent class, store info is the message of Agent class, Inventory manage rule is the rule of operating its knowledge.

According to the structure of inventory management Agent and the principles of planning a single Agent, a single Agent of system constructed to a class and the behaviors of Agent organized to multiple classes based on requirement. In an UML class view, behaviors of an Agent can be used by others, and an Agent can also have multiple behaviors. The class view based on UML of Agent oriented distributed inventory management system is shown in Figure 2. The relationship between Agent in the figure represents their communication.

\section{CONCLUSION}

Agent has the characteristics of autonomy, sociality, responsiveness, and currently there is CASE tools provide functions that map the model established with UML to practical programming language code in order to realize modeling specification of Agent oriented model and convert the abstract model into specific operation system through compile in the implementation phase.

Therefore, this paper adopts visual modeling language of UML to construct an Agent oriented inventory management system model, and provides an effective method for inventory management.

\section{Acknowledgements}

This work was financially supported by the Guangdong Natural Science Foundation (10151032001000001).

\section{References}

[1] Xiao Renbin, Hu Bin, Gong Xiaoguang, Management System Simulation, Beijing: Press of Electronics Industry, 2008.

[2] Xia Liling, The distributed inventory management model based on Intranet. Journal of nanjing industry professional technology institute.20121671-4644 (2012) 02-0047-03.

[3] Li Xiujuan, Li Juan, UML oriented modeling method based on UMAM, Science and Technology innovation Forum, 20101671 - 7597 (2010) 0820188 - 2.

[4] Qiao Dongping, Li Hao, Xiao Yanqiu, Agent oriented distributed production management system modeling, Manufacturing Automation, 20131009-0134 (2013)08 (last) -0077-05.

[5] Wang Minghui, Zhang Shusheng, Zhou Jingtao, Zhao Han, An Agent modeling method based on UML, http:/wenku.baidu.com/view/92919b0c7cd184254b35358c.html

[6]Qian Yue, The design and implementation of distributed library management system based on Agent, Journal of Shangqiu Normal University, 2011672, 3600 (2011): 03 - 0074 - 5

[7] Xie Xingxing, Liu Xiaosong, Wang Jianning, UML unified modeling course and experiment guide, Beijing: Tsinghua University Press, 2013,1-1.

[8] Jiang Meiyun, Agent oriented modeling based on UML, Software Tribune, 2011672-7800 (2011) 011-0003-02.

[9] Liu Dan, Cao Jiantong, Chen Jianming, Application of modeling based on Agent: overview of research, Technology and Economy, 20141002-980X (2014) 11-0096-07.

[10] Fu Yu, Xu Tao, Wang Hong, Ding building, Intelligent Agent oriented modeling of complex distributed systems, Journal of Beijing University of Technology, 20110254, 0037 (2011): 06 - 0955 6. 
[11] Lu Qing, Hu Fei, The design of distributed inventory management system and its inventory module, Microelectronics and Computer, 20051000-7180 (2005) 01-017-03.

[12] Ceng Liming, Han Ruizhu, Simulation of distributed inventory management system,Computer Integrated Manufacturing Systems, 1006-5911 (2010) 05-1067-07. 